\title{
Distinct DNA Methylomes of Human Placentas Between Pre-Eclampsia and Gestational Diabetes Mellitus
}

\author{
Lan Liua,b Xiang Zhang ${ }^{\mathrm{b}}$ Can Rong ${ }^{\mathrm{a}}$ Can Rui ${ }^{\mathrm{b}}$ Hui Ji ${ }^{\mathrm{b}}$ Yu-jia Qian ${ }^{\mathrm{b}}$ Ruizhe Jia ${ }^{\mathrm{b}}$ \\ Lizhou Sun ${ }^{c}$ \\ ${ }^{a}$ Nanjing Maternal and Child Health Medical Institute, Nanjing Medical University Affiliated Nanjing \\ Maternal and Child Health Hospital, bDepartment of Obstetrics, Nanjing Medical University Affiliated \\ Nanjing Maternal and Child Health Hospital, 'Department of Gynecology and Obstetrics, \\ First Affiliated Hospital of Nanjing Medical University, Nanjing, China
}

\section{Key Words}

DNA methylation $•$ Placenta $•$ Pre-eclampsia $\bullet$ Gestational diabetes mellitus

\begin{abstract}
Background: The placenta acts not only as a conduit of nutrient and waste exchange between mother and developing fetus but also functions as a regulator of the intrauterine environment. Pre-eclampsia (PE) and gestational diabetes mellitus (GDM) are leading causes of complications during pregnancy. Pathophysiologies show that they are associated with one another. Epigenetics provides a link between environmental factors that have previously been linked to poor pregnancy outcomes and fetal programming. Methods and Results: The present study investigated genome-wide DNA methylation changes in PE and GDM compared with control subjects through DNA methylation microarray. We found that the methylation patterns of placentas from PE and GDM women were similar; $64.4 \%$ of the annotated genes with differential methylation presented concordant changes between PE and GDM patients. Significantly, the same functional processes were affected by PE and GDM, with cell adhesion and cell differentiation being the most populated clusters and including genes related to carbohydrate metabolism and lipid metabolism. Conclusion: Our work showed that of DNA methylation patterns in human placentas are reliably and significantly associated with PE and GDM. DNA methylation status in the human placenta can function as a marker for the intrauterine environment and potentially play a functional role in PE and GDM development.
\end{abstract}

L. Liu and X. Zhang contributed equally to this work.

Ruizhe Jia

and Lizhou Sun
Department of Obstetrics, Nanjing Medical University Affiliated Nanjing Maternal and Child Health Hospital, Nanjing 210004 (China); and Department of Gynecology and Obstetrics, First Affiliated Hospital of Nanjing Medical University, Nanjing 210029 (China)

E-Mail cuixianwei198615@163.com and E-Mail lizhou_sun121@hotmail.com 


\section{Introduction}

Pre-eclampsia (PE) and gestational diabetes mellitus (GDM) are leading causes of complications during pregnancy with serious negative effects on the health of both the mother and her unborn child [1-3]. The pathophysiologies of both illnesses have been shown to be associated with each other [3-6]. It appears that gestational diabetes and pre-eclampsia have several mechanisms in common: altered carbohydrate metabolism causes vascular changes such as arteriosclerosis and a glomerular filtration dysfunction, which results in a predisposition to pre-eclampsia. Some authors have recently discussed gestational diabetes as a risk factor of pre-eclampsia and pre-eclampsia as a possible consequence of gestational diabetes [5]. This theory is supported by a prospective random study that the treatment of gestational diabetes mellitus reduced the rate of pre-eclampsia by $30 \%$ [7]. Other authors cite dysfunctions such as increased insulin resistance as the cause of both illnesses $[3,4,8,9]$. Several authors therefore have reached the conclusion that the state of research is inadequate and ambiguous [4, 8-10] for the difficulty of providing support for these explanations in the form of epidemiological data.

Epigenetics constitutes an important mechanism capable of regulating gene transcription, linking early life events to adult morbidity. Epigenetics entails heritable changes in chromatin that alter gene expression without changing the DNA sequence [11, 12]. DNA methylation is the most commonly studied epigenetic processes which is subject to developmental and environmental regulation and potentially modifiable with appropriate interventions [13]. It is primarily observed in $\mathrm{CpG}$ dinucleotides, which are underrepresented in the genome, generally at low density, but may be enriched in the promoter regions of genes $[14,15]$. Elevated DNA methylation in such regions is generally associated with gene inactivity whereas a lack of methylation indicates the potential for gene expression.

The placenta function is directly linked to fetal development, and as such, placental dysfunction is implicated in many pregnancy-associated diseases [16]. Aberrant placental gene expression has been linked to pre-eclampsia, intrauterine growth restriction, gestational diabetes mellitus and gestational trophoblastic disease [17-23]. Given the link between gene expression and promoter methylation, it is reasonable to assume that aberrant expression in placental disease is associated with a disrupted DNA methylation profile or other epigenetic change [24]. Furthermore, epigenetics may provide a link between environmental factors that have previously been linked to poor pregnancy outcomes and fetal programming [25, 26]. It is reported that the patterns of genome-wide hypermethylation in promoters are widespread in the chorioamniotic membranes of early onset pre-eclampsia [27] and DNA methylome profiling of maternal peripheral blood and placentas reveal potential fetal DNA markers [28].

The critical role of placenta in responding to intrauterine environment and the environment can influence the pattern of DNA methylation. The present study investigated genome-wide DNA methylation changes of pre-eclampsia and gestational diabetes mellitus to examine their association in human placentas. We found that the overall methylation patterns of placentas from PE and GDM women were similar, 64.4\% (3348/5195) of the annotated genes presented concordant methylation changes. Additionally, several genes previously associated with carbohydrate metabolism with concordant methylation alterations were identified. This study will be helpful in exploring gestational diabetes mellitus and preeclampsia's having several mechanisms in common from the perspective of epigenetics.

\section{Material and Methods}

\section{Human Samples}

Human placental tissues were collected at the Maternal and Child Health Hospital (Nanjing, Jiangsu, China) with the approval of Nanjing Medical University, Human Research Ethics Committee. Patients were fully informed of the study and signed informed consent forms of their own accord. Sometimes these 
puerperas were in an extremely dangerous situation (such as in a state of unconsciousness) and required immediate surgery. In this situation, consent to collect placental tissues was obtained from a family member instead of the patient. After that, we sought the patients' medical records with the consent of the patients. Placental biopsies were obtained during Cesarean section (CS) from both normal patients $(n=30)$ and those with pre-eclampsia $(n=27)$ or gestational diabetes mellitus $(n=28)$. Pre-eclampsia was defined as a blood pressure of $\geq 140 / 90 \mathrm{mmHg}$ taken twice, 6 hours apart, with proteinuria of $\geq 2+$ or $\geq 300 \mathrm{mg}$ in a 24hour collection. Women were diagnosed with GDM when a $75 \mathrm{~g}$ oral glucose tolerance test (OGTT) revealed either a fasting venous plasma glucose level of $5.1 \mathrm{mmol} / \mathrm{L}$ glucose and/or at 2 hours a plasma glucose level $>8.1 \mathrm{mmol} / \mathrm{L}$ glucose. Pre-eclampsia and gestational diabetes mellitus patients were control-matched with uncomplicated term pregnancies with normotensive subjects and normal glucose tolerance (NGT). Information on maternal reproductive data, delivery characteristics, and infant outcomes were collected from maternal medical records and presented in Table 1.

Placentas were prepared within 10-15 minutes of delivery. Tissues were dissected and repeatedly rinsed until free from blood in chilled PBS $\left(4^{\circ} \mathrm{C}\right)$. Placental lobules were always taken from the central region of the placenta, and the basal plate and chorionic surface were removed with the villous tissue obtained from the middle cross section. Dissected tissue was immediately snap frozen in liquid nitrogen and stored at $-80^{\circ} \mathrm{C}$ until ready for DNA extraction.

\section{DNA extraction and modification}

DNA was extracted from the placenta samples using the QIAmp tissue kit (Qiagen, Hilden, Germany) following manufacturer's protocols. Purified DNA was quantified with the NanoDrop ND1000 spectrophotometer, and $1 \mu \mathrm{g}$ of placental DNA was bisulfite modified using the EZ DNA ethylation Kit D5008 (Zymogen, \# D5001).

\section{DNA methylation microarray}

DNA from placenta samples was fragmented by sonication to 300-1000 bp average size as determined on agarose gels. Enrichment of the methylated double-stranded DNA fraction by MIRA was performed as described previously $[29,30]$. The labeling of amplicons, microarray hybridization, and scanning were performed according to the NimbleGen protocol. NimbleGen tiling arrays were used for hybridization (385 K Human CpG Island plus Promoter arrays). The single array design covers all 28,226 University of California, Santa Cruz (UCSC) Genome Browser-annotated CpG islands and the promoter regions for all RefSeq genes. The promoter region covered is $1 \mathrm{~kb}(-800$ to +200 relative to the transcription start sites). For all the samples, the MIRA-enriched DNA was compared with the input DNA. We conducted three technical replicates for each enrichment and hybridization, after which we averaged the log ratios for each replicate to produce one value per individual per locus.

\section{Microarray Data Preprocessing}

The Illumina GenomeStudio Software (version 2011.1) was used to assess quality and extract the DNA methylation signals from scanned arrays. Methylation data were extracted as raw signals with no background subtraction, and the data were normalized to control probes present on the array. Log 2 ratio data were converted into P-value scores using Student's $t$-test. Probes were selected as positive if their Pvalue scores were above $2(\mathrm{P}<0.05)$. In addition, the multivariate characteristics (or Mahalanobis distance based on fitted mean vector and variance-covariance matrix) of array control probes supplied by NimbleGen were used to diagnose problems such as poor bisulfite conversion or color-specific problems; none were identified.

\section{Gene Ontology (GO) and pathway analysis}

We next used Gene Ontology analysis to investigate if any common functional trends are associated with the genes exhibiting differences from the PE and GDM groups compared with the control group. Those loci exhibiting a significance value of less than $\mathrm{p}<0.05$ from the Student's $t$-test were selected to include only those loci likely to demonstrate a true DNA-methylation difference between groups. We then mapped all gene candidates to GO terms in the database (http://www.geneontology.org/), calculating gene numbers for each term. Gene networks and canonical pathways representing key genes were identified using the curated IPA (Ingenuity Pathway Analysis) database according to KEGG, Biocarta, and Reatome, as previously 
Table 1. Clinical characteristics of PE, GDM and control women. ${ }^{\mathrm{a}} \mathrm{p}<0.05$ when compared with control group. ${ }^{\mathrm{b}} \mathrm{p}<0.001$ when compared with control group. BMI, body mass index; BP, blood pressure; SD, standard deviation

\begin{tabular}{lccc}
\hline Cliniacl Features & PE $\mathrm{n}=27$ & $\mathrm{GDM} \mathrm{n}=28$ & Control $\mathrm{n}=30$ \\
\hline Maternal age $($ mean $\pm \mathrm{SD})$ & $30.1 \pm 2.7$ & $30.8 \pm 1.4$ & $29.7 \pm 1.8$ \\
Maternal BMI $($ mean $\pm \mathrm{SD})$ & $32 \pm 2.9^{\mathrm{a}}$ & $31.3 \pm 0.6^{\mathrm{a}}$ & $26.3 \pm 1.9$ \\
Week gestation $($ mean $\pm \mathrm{SD})$ & $35.7 \pm 0.9^{\mathrm{a}}$ & $36.2 \pm 0.8^{\mathrm{a}}$ & $36.4 \pm 0.5$ \\
Mode of devliviery CS(\%) & $100 \%$ & $100 \%$ & $100 \%$ \\
Brith weight $(\mathrm{g})(\mathrm{mean} \pm \mathrm{SD})$ & $2112.0 \pm 454.3^{\mathrm{b}}$ & $3920.5 \pm 54.3^{\mathrm{a}}$ & $3620.5 \pm 26.9$ \\
BP Systolic $(\mathrm{mmHg})$ & $151.3 \pm 12.1^{\mathrm{a}}$ & $127.8 \pm 7.1$ & $117.6 \pm 5.6$ \\
BP Diastolic $(\mathrm{mmHg})$ & $95.2 \pm 5.6^{\mathrm{a}}$ & $80.3 \pm 5.4$ & $78.6 \pm 2.1$ \\
Proteinuria $(\mathrm{g} / 24 \mathrm{~h})$ & $5.3 \pm 2.5$ & $\mathrm{~N} / \mathrm{A}$ & $\mathrm{N} / \mathrm{A}$ \\
OGTT fasting & $4.9 \pm 0.5$ & $5.9 \pm 0.3$ & $4.7 \pm 0.2$ \\
1h & $6.8 \pm 1.2$ & $10.5 \pm 0.3^{\mathrm{b}}$ & $5.3 \pm 0.3$ \\
$2 \mathrm{~h}$ & $5.0 \pm 0.7$ & $8.6 \pm 0.4^{\mathrm{b}}$ & $4.9 \pm 0.5$ \\
\hline
\end{tabular}

described [31]. Those loci exhibiting a significance value of less than $\mathrm{p}<0.05$ from the Student's $t$-test were selected to include only those loci likely to demonstrate a true DNA-methylation difference between groups. The threshold of significance was defined by the P-value and FDR.

\section{Validation of the microarray finding}

We further investigated samples from 85 placentas by bisulfite sequencing PCR (BSP), including 30 normal placentas, 28 placentas from women with GDM and 27 placentas from women with PE. Primers were designed with MethPrimer using the loci nominated from microarray analyses. The four pair primers were as follow: Resistin (Forward: TATTGTTTGTTTAGGGGTTTTTTTT, Reverse: AAAAACAAC TATCACTTACCCTCTC, 163 bp), RBP4 (Forward: TGTATAAGTGGATTTTAGA AGGTAGA, Reverse: ACCCTAACTCAATTACCC TACACTC, 226 bp), GLUT3 (Forward: TAAGGGAAATGTTTTATTTTTTGAG, Reverse: CATAAAACAACCA CCAATAACAAC, 192 bp), PPAR $\alpha$ (Forward: GGTTTAGTGGTGTTTTTGTGTA GAT, Reverse: TCAAACTCCTAACTTCAAATAATCC, $165 \mathrm{bp})$.

PCR amplifications were performed with a standard hot-start PCR protocol in $25 \mu$ l volume reactions containing $3 \mu \mathrm{l}$ of sodium-bisulfitetreated DNA, $1 \mathrm{mM}$ primers, and a master mix containing hot-start Taq polymerase (Sigma). PCR conditions were as follows: $95^{\circ} \mathrm{C}$ for $10 \mathrm{~min}, 40$ cycles $\left(95{ }^{\circ} \mathrm{C} / 40 \mathrm{~s} ; 50^{\circ} \mathrm{C} / 40 \mathrm{~s}\right.$; $72{ }^{\circ} \mathrm{C} / 40 \mathrm{~s}$ ), and then $72{ }^{\circ} \mathrm{C}$ for $7 \mathrm{~min}$. All PCR reactions were checked on a $1.5 \%$ agarose gel to ensure successful amplification. For BSP clone sequence analysis, the PCR products were subcloned into a pMD-19-T vector (Takara Bio, Inc., Japan). Ten to twenty clones were selected for sequencing to identify methylation differences between PE, GDM and control groups.

Statistical analysis.

The statistical significance of the methylation datasets was performed using a one-way ANOVA corrected for multiple comparisons followed by the Student's $t$-test with a significance of $\mathrm{p}<0.05$. Data from the bisulfite sequencing PCR experiments were analyzed with the Student's unpaired or paired $t$-tests where appropriate. Values are presented as mean \pm standard deviation.

\section{Results}

\section{Maternal and neonatal characteristics}

Table 1 describes the maternal and infant characteristics of the randomly generated training and testing datasets. All recruited women belonged to the same age group, however, the body mass index of patients from PE and GDM groups were significant different from control group. The duration of gestation was significantly lower in the control group when compared with the pre-eclampsia group and the gestational diabetes mellitus group. The systolic and diastolic blood pressure of pre-eclampsia women was significantly higher than normotensive women. Further, baby weight was significantly higher in the gestational diabetes mellitus group when compared with the control group. GDM and PE women had a 
Table 2. Distribution of differentially methylated regions in placentas DNA from PE and GDM. Differential methylation analysis threshold included probes with detection $\mathrm{P}$ value $<$ 0.001. aPercentage of DMR from the average number of array probes detected per PE sample at $p<0.001(185,577)$. bPercentage of DMR from the average number of array probes detected per GDM sample at $\mathrm{p}<0.001(185,554)$. *Probes showing gain or loss of DNA methylation in PE and GDM samples comparison to controls. "DM probes excluding tho-

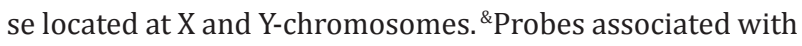
annotated genes. ${ }^{\dagger}$ Probes associated with annotated genes showing concordant changes in methylation in both PE and GDM samples

\begin{tabular}{lll}
\hline Gene name & PE & GDM \\
\hline Differentially methylated regions & $8191^{\text {a }}$ & $10424^{\mathrm{b}}$ \\
Increased methylation* $^{*}$ & 3394 & 4021 \\
Located in Autosomal CHR $^{*}$ & 272 & 279 \\
Annotated genes\& $^{*}$ & 1738 & 2021 \\
Co-variation $^{\dagger}$ & 1532 & \\
Decreased methylation $^{*}$ & 4797 & 6403 \\
Located in Autosomal CHR $^{*}$ & 431 & 269 \\
Annotated genes\& $^{*}$ & 2140 & 2644 \\
Co-variation $^{\dagger}$ & 1816 & \\
\hline
\end{tabular}

A

C

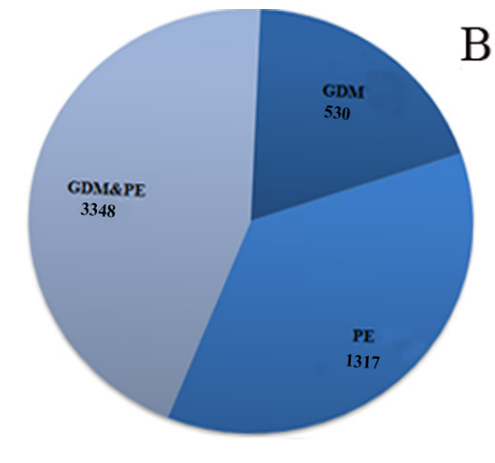

B

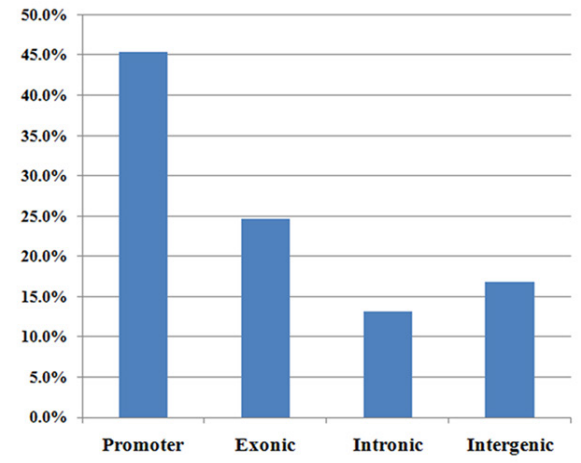

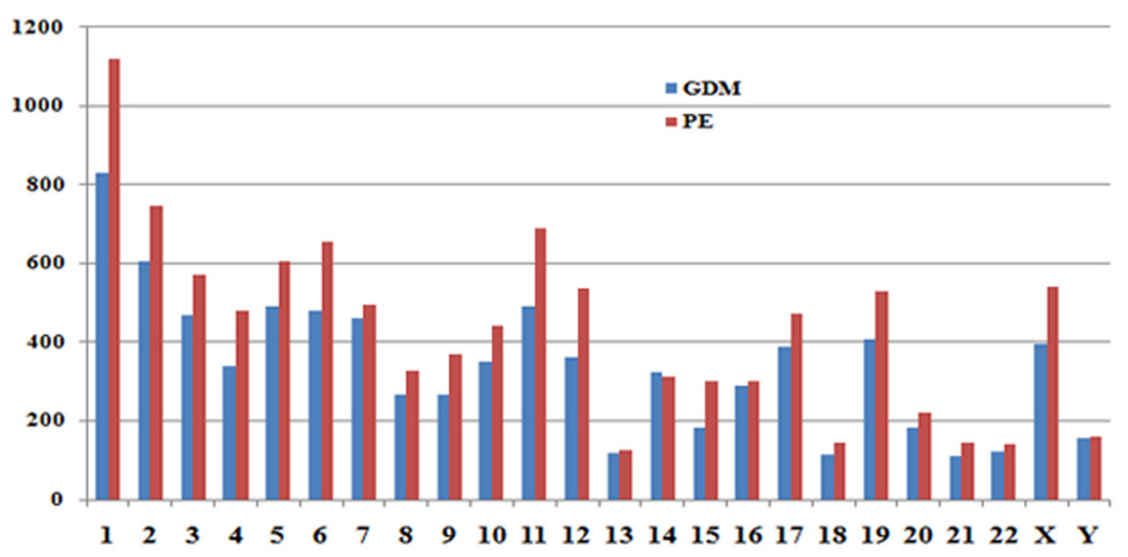

Fig. 1. (A) Comparison of the fractions of consistent and inconsistent methylated loci in PE and GDM samples. (B) The breakdown of the Methyl-seq regions into specific annotated genomic elements and binding locations. Coordinates for promoters and other genic elements were calculated using the transcription start sites and gene annotations from the Known Genes file, compiled by the UCSC Genome Browser. (C) Chromosome location distribution of differentially methylated regions in PE and GDM samples. GDM, hypemethylated and hypomethylated regions of GDM placentas. PE-up, hypemethylated and hypomethylated regions of PE placentas.

significantly greater BMI at their first antenatal visit (12 weeks gestation) when compared with the control group. Although fasting OGTT levels were not significantly different between groups, both 1-hour and 2-hour OGTT readings were significantly higher for GDM women. It should be noted that we did not exclude the influence of BMI, whether it has some effects is still unknown. 
Liu et al.: DNA Methylomes of PE and GDM Patients' Placentas

Table 3. Differentially methylated autosomal genes that co-varied in placentas DNA from PE and GDM

\begin{tabular}{|c|c|c|c|c|c|c|c|}
\hline $\begin{array}{l}\text { Chromosome } \\
\text { Methylated re }\end{array}$ & \multicolumn{6}{|c|}{ Methylated regions both down-regulated in placentas DNA from PE and GDM ( Both $>3$ folds) } & Gene name \\
\hline chr7 & 104696526 & 104697491 & -4 & $5.3 \mathrm{E}-05$ & -4.26 & $2.3 \mathrm{E}-05$ & SRPK2 \\
\hline chr5 & 76283637 & 76284586 & -3 & $7.4 \mathrm{E}-03$ & -4.12 & $3.4 \mathrm{E}-03$ & CRHBP \\
\hline $\operatorname{chr} 12$ & 91345874 & 91346517 & -3.37 & $9.8 \mathrm{E}-16$ & -4.08 & $4.8 \mathrm{E}-16$ & CLLU1OS \\
\hline $\operatorname{chr} 11$ & 55757093 & 55757994 & -3.88 & $2.8 \mathrm{E}-02$ & -4.06 & $1.8 \mathrm{E}-02$ & OR5T2 \\
\hline $\operatorname{chr} 13$ & 45577339 & 45577921 & -3.4 & $4.2 \mathrm{E}-03$ & -4.06 & $3.2 \mathrm{E}-03$ & CPB2 \\
\hline $\operatorname{chr} 12$ & 7487837 & 7488584 & -4.05 & $2.3 \mathrm{E}-02$ & -4.05 & $2.5 \mathrm{E}-02$ & CD163L1 \\
\hline $\operatorname{chr} 12$ & 101036295 & 101037102 & -3.28 & $1.5 \mathrm{E}-05$ & -3.86 & 2.5E-05 & NUP37 \\
\hline chr3 & 162421057 & 162421765 & -3.04 & 2.3E-03 & -3.75 & 5.3E-03 & RBP4 \\
\hline $\operatorname{chr} 1$ & 246880827 & 246881562 & -3.43 & $4.9 \mathrm{E}-02$ & -3.73 & $3.9 \mathrm{E}-02$ & OR $2 \mathrm{~T} 27$ \\
\hline $\operatorname{chr} 2$ & 167700164 & 167700866 & -3.28 & $2.7 \mathrm{E}-03$ & -3.7 & $2.7 \mathrm{E}-03$ & CMYA3 \\
\hline $\operatorname{chr} 12$ & 53809144 & 53809873 & -3.21 & $4.5 \mathrm{E}-03$ & -3.66 & $4.5 \mathrm{E}-03$ & OR9K2 \\
\hline $\mathrm{chr} 2$ & 204509011 & 204509714 & -4.26 & $1.1 \mathrm{E}-09$ & -3.63 & $5.3 \mathrm{E}-04$ & Resistin \\
\hline chr6 & 99969288 & 99969995 & -3.22 & 5.3E-05 & -3.62 & $7.4 \mathrm{E}-03$ & C6orf111 \\
\hline chr3 & 161599340 & 161600476 & -3.29 & $7.4 \mathrm{E}-03$ & -3.59 & $9.8 \mathrm{E}-10$ & IFT80 \\
\hline chr6 & 28199585 & 28200288 & -5.32 & $9.8 \mathrm{E}-13$ & -3.57 & $2.8 \mathrm{E}-02$ & ZNF435 \\
\hline $\operatorname{chr} 11$ & 18018686 & 18019564 & -3.74 & $2.8 \mathrm{E}-02$ & -3.45 & $4.2 \mathrm{E}-03$ & TPH1 \\
\hline chr6 & 26127898 & 26128552 & -3.59 & $3.2 \mathrm{E}-03$ & -3.41 & $3.5 \mathrm{E}-02$ & PPAR $\alpha$ \\
\hline $\operatorname{chr} 11$ & 5835631 & 5836283 & -3.32 & $2.5 \mathrm{E}-02$ & -3.36 & $2.5 \mathrm{E}-05$ & OR52E8 \\
\hline chr6 & 27399778 & 27400622 & -3.08 & $4.5 \mathrm{E}-05$ & -3.36 & $5.8 \mathrm{E}-03$ & FKSG83 \\
\hline $\operatorname{chr} 2$ & 113451639 & 113452228 & -3.12 & $5.3 \mathrm{E}-03$ & -3.34 & $4.9 \mathrm{E}-03$ & IL1F9 \\
\hline $\operatorname{chr} 6$ & 64339091 & 64340035 & -3.78 & $4.9 \mathrm{E}-02$ & -3.33 & $2.7 \mathrm{E}-03$ & PTP4A1 \\
\hline chr6 & 50124184 & 50125095 & -3.75 & $4.2 \mathrm{E}-03$ & -3.3 & $2.9 \mathrm{E}-03$ & DEFB112 \\
\hline chr13 & 48854948 & 48855791 & -3.16 & $5.3 \mathrm{E}-05$ & -3.3 & $4.3 \mathrm{E}-05$ & CAB39L \\
\hline chr2 & 132099568 & 132100319 & -3.37 & $7.4 \mathrm{E}-03$ & -3.28 & 5.3E-05 & FKSG30 \\
\hline $\operatorname{chr} 3$ & 115494779 & 115495265 & -3.53 & $9.8 \mathrm{E}-16$ & -3.27 & 7.4E-04 & VSIG9 \\
\hline $\operatorname{chr} 15$ & 40288057 & 40288571 & -3.7 & $2.8 \mathrm{E}-02$ & -3.24 & $9.8 \mathrm{E}-16$ & VPS39 \\
\hline chr1 & 78887319 & 78888224 & -4.15 & $4.2 \mathrm{E}-03$ & -3.19 & $2.8 \mathrm{E}-02$ & IFI44 \\
\hline chr7 & 113346120 & 113346824 & -3.33 & $2.5 \mathrm{E}-02$ & -3.19 & $4.2 \mathrm{E}-03$ & PPP1R3A \\
\hline $\operatorname{chr} 16$ & 30364923 & 30365414 & -3.22 & $2.5 \mathrm{E}-05$ & -3.18 & $2.5 \mathrm{E}-02$ & SEPHS2 \\
\hline $\operatorname{chr} 14$ & 23982151 & 23982604 & -3.81 & $5.2 \mathrm{E}-03$ & -3.16 & $2.5 \mathrm{E}-05$ & C14orf 124 \\
\hline $\operatorname{chr} 4$ & 177427177 & 177427991 & -3.01 & $4.9 \mathrm{E}-02$ & -3.15 & $5.3 \mathrm{E}-04$ & ASB5 \\
\hline chr4 & 177427177 & 177427991 & -3.01 & $2.7 \mathrm{E}-03$ & -3.15 & $4.9 \mathrm{E}-02$ & ASB5 \\
\hline $\operatorname{chr} 11$ & 6147947 & 6148797 & -3.6 & $4.5 \mathrm{E}-03$ & -3.14 & 2.7E-03 & OR52B2 \\
\hline $\operatorname{chr} 18$ & 22699776 & 22700480 & -3.4 & $1.1 \mathrm{E}-09$ & -3.12 & $3.5 \mathrm{E}-03$ & AQP4 \\
\hline chr4 & 2200579 & 2201234 & -3.08 & $1.1 \mathrm{E}-12$ & -3.12 & 1.3E-09 & POLN \\
\hline $\operatorname{chr} 12$ & 47162495 & 47163207 & -3.05 & $3.7 \mathrm{E}-06$ & -3.12 & $3.7 \mathrm{E}-06$ & C12orf54 \\
\hline chr18 & 50408694 & 50409566 & -4.21 & $3.3 \mathrm{E}-05$ & -3.11 & $1.3 \mathrm{E}-05$ & C18orf26 \\
\hline $\operatorname{chr} 14$ & 64002798 & 64003646 & -3.82 & $1.3 \mathrm{E}-06$ & -3.11 & $1.4 \mathrm{E}-07$ & AKAP5 \\
\hline chr1 & 196874047 & 196874821 & -3.51 & $2.4 \mathrm{E}-03$ & -3.1 & $2.7 \mathrm{E}-04$ & PTPRC \\
\hline $\operatorname{chr} 11$ & 62813206 & 62814061 & -4.15 & $2.7 \mathrm{E}-04$ & -3.09 & $1.5 \mathrm{E}-04$ & SLC22A10 \\
\hline chr1 & 156954378 & 156954990 & -3.53 & $3.7 \mathrm{E}-04$ & -3.08 & $2.4 \mathrm{E}-04$ & OR6K3 \\
\hline $\operatorname{chr} 3$ & 166278846 & 166279697 & -3.26 & $1.4 \mathrm{E}-04$ & -3.08 & 1.4E-04 & SI \\
\hline $\operatorname{chr} 14$ & 22634682 & 22635284 & -3.02 & $1.5 \mathrm{E}-06$ & -3.07 & $3.5 \mathrm{E}-05$ & ACIN1 \\
\hline chr3 & 44728343 & 44728813 & -3.23 & $1.4 \mathrm{E}-06$ & -3.05 & $2.4 \mathrm{E}-05$ & ZNF502 \\
\hline chr11 & 4563831 & 4564388 & -2.62 & $5.4 \mathrm{E}-05$ & -3.05 & $8.4 \mathrm{E}-05$ & OR52I2 \\
\hline chr7 & 133461864 & 133462545 & -3.43 & $1.5 \mathrm{E}-05$ & -3.04 & $2.5 \mathrm{E}-06$ & FLJ32786 \\
\hline chr11 & 55362024 & 55362958 & -3.15 & $1.3 \mathrm{E}-07$ & -3.04 & $1.4 \mathrm{E}-06$ & OR5D16 \\
\hline $\operatorname{chr} 10$ & 69126045 & 69126688 & -4.09 & $2.5 \mathrm{E}-05$ & -3.02 & $1.5 \mathrm{E}-04$ & CTNNA3 \\
\hline $\operatorname{chr} 12$ & 10174035 & 10174905 & -3.37 & $1.7 \mathrm{E}-07$ & -3.02 & 3.7E-06 & CLEC7A \\
\hline $\operatorname{chr} 1$ & 157675789 & 157676325 & -3.76 & 3.3E-05 & -3.01 & $1.3 \mathrm{E}-06$ & OR10J1 \\
\hline chr8 & 69511912 & 69512817 & -3.63 & $1.7 \mathrm{E}-07$ & -3 & 1.3E-05 & C8orf34 \\
\hline \multicolumn{8}{|c|}{ Methylated regions both up-regulated in placentas DNA from PE and GDM ( Both $>4$ folds) } \\
\hline chr16 & 2154227 & 2156176 & 4.68 & 2.7E-05 & 4.03 & 3.7E-06 & TRAF7 \\
\hline chr8 & 1720479 & 1721321 & 4.4 & $1.3 \mathrm{E}-05$ & 4.08 & $3.3 \mathrm{E}-04$ & C8orf61 \\
\hline chr5 & 140241262 & 140242338 & 3.66 & $2.7 \mathrm{E}-04$ & 4.17 & $1.5 \mathrm{E}-04$ & PCDHA13 \\
\hline $\operatorname{chr} 2$ & 72227038 & 72227749 & 4.37 & $3.3 \mathrm{E}-04$ & 4.2 & $3.4 \mathrm{E}-05$ & GLUT3 \\
\hline $\operatorname{chr} 22$ & 18969719 & 18970878 & 4.16 & $1.4 \mathrm{E}-04$ & 4.22 & $1.4 \mathrm{E}-04$ & LOC643069 \\
\hline chr10 & 135000492 & 135001242 & 4.18 & $5.4 \mathrm{E}-05$ & 4.27 & $1.5 \mathrm{E}-05$ & DRD1IP \\
\hline $\operatorname{chr} 11$ & 1891292 & 1893649 & 4.2 & $1.4 \mathrm{E}-05$ & 4.28 & $2.4 \mathrm{E}-05$ & TNNT3 \\
\hline chr8 & 86753857 & 86755646 & 4.54 & $6.4 \mathrm{E}-05$ & 4.34 & 8.4E-05 & REXO1L3P \\
\hline $\operatorname{chr} 7$ & 72051109 & 72051768 & 4.02 & $1.5 \mathrm{E}-06$ & 4.38 & $2.5 \mathrm{E}-06$ & POM121 \\
\hline $\operatorname{chr} 21$ & 13974048 & 13975959 & 4.46 & $1.5 \mathrm{E}-06$ & 4.38 & $1.4 \mathrm{E}-06$ & LOC441956 \\
\hline chr8 & 86908165 & 86910007 & 4.63 & $2.5 \mathrm{E}-03$ & 4.45 & $2.6 \mathrm{E}-03$ & REX01L1 \\
\hline $\operatorname{chr} 2$ & 853943 & 855102 & 5.07 & $2.7 \mathrm{E}-03$ & 4.62 & 2.7E-03 & LOC642837 \\
\hline chr11 & 1849454 & 1850493 & 4.36 & 2.7E-06 & 4.68 & $3.7 \mathrm{E}-06$ & LSP1 \\
\hline $\operatorname{chr} 15$ & 18823777 & 18824438 & 4.79 & $3.5 \mathrm{E}-05$ & 4.68 & $3.3 \mathrm{E}-05$ & LOC646122 \\
\hline chr8 & 86871871 & 86873718 & 4.67 & $2.3 \mathrm{E}-07$ & 4.69 & $1.3 \mathrm{E}-07$ & REX01L1 \\
\hline $\operatorname{chr} 10$ & 126906574 & 126907323 & 4.11 & $2.4 \mathrm{E}-03$ & 4.88 & 2.7E-03 & LOC642622 \\
\hline $\operatorname{chr} 3$ & 140246279 & 140247062 & 4.24 & $1.5 \mathrm{E}-04$ & 4.9 & $2.5 \mathrm{E}-04$ & FLJ46210 \\
\hline chr8 & 86741932 & 86743791 & 4.43 & $1.7 \mathrm{E}-06$ & 4.91 & $3.7 \mathrm{E}-06$ & LOC653760 \\
\hline $\operatorname{chr} 15$ & 33172135 & 33172784 & 4.54 & $4.3 \mathrm{E}-05$ & 4.92 & 3.3E-05 & LOC 441722 \\
\hline chr8 & 86884061 & 86885908 & 4.97 & $1.3 \mathrm{E}-07$ & 4.94 & $5.3 \mathrm{E}-07$ & REX01L2P \\
\hline $\operatorname{chr} 19$ & 3788785 & 3789439 & 4.92 & $2.4 \mathrm{E}-03$ & 4.98 & $2.9 \mathrm{E}-03$ & KIAA1086 \\
\hline chr12 & 129937787 & 129938451 & 4.73 & $2.9 \mathrm{E}-04$ & 5.06 & $2.5 \mathrm{E}-04$ & LOC390367 \\
\hline chr8 & 86760698 & 86762541 & 4.86 & $3.4 \mathrm{E}-05$ & 5.07 & $3.6 \mathrm{E}-04$ & REX01L1 \\
\hline chr6 & 101018081 & 101018925 & 4.96 & $1.4 \mathrm{E}-04$ & 5.07 & 3.7E-06 & SIM1 \\
\hline $\operatorname{chr} 21$ & 44030904 & 44031563 & 4.91 & 5.5E-05 & 5.14 & $3.3 \mathrm{E}-05$ & $\mathrm{D} 21 \mathrm{~S} 2056 \mathrm{E}$ \\
\hline $\operatorname{chr} 6$ & 150327197 & 150328184 & 4.61 & $2.4 \mathrm{E}-05$ & 5.22 & $1.3 \mathrm{E}-07$ & ULBP1 \\
\hline chr8 & 6411613 & 6412267 & 4.82 & $8.4 \mathrm{E}-05$ & 5.27 & $2.4 \mathrm{E}-03$ & ANGPT2 \\
\hline $\operatorname{chr} 1$ & 1254852 & 1255501 & 5.1 & $2.5 \mathrm{E}-06$ & 5.32 & $2.5 \mathrm{E}-04$ & TAS1R3 \\
\hline chr19 & 618079 & 618738 & 5.13 & $1.4 \mathrm{E}-06$ & 5.43 & $3.4 \mathrm{E}-04$ & RNF126 \\
\hline $\operatorname{chr} 17$ & 78606128 & 78606792 & 3.88 & $1.5 \mathrm{E}-04$ & 5.47 & $3.7 \mathrm{E}-06$ & B3GNTL1 \\
\hline $\operatorname{chr} 16$ & 87759722 & 87760446 & 5.46 & $2.4 \mathrm{E}-04$ & 5.49 & $3.4 \mathrm{E}-04$ & LOC400558 \\
\hline $\operatorname{chr} 18$ & 75188453 & 75189122 & 5.56 & $2.5 \mathrm{E}-03$ & 5.57 & $2.5 \mathrm{E}-03$ & АТР9В \\
\hline $\operatorname{chr} 17$ & 77870432 & 77871156 & 5.89 & 2.7E-03 & 5.85 & 2.7E-03 & CD7 \\
\hline $\operatorname{chr} 6$ & 31026748 & 31027714 & 4.95 & $1.1 \mathrm{E}-19$ & 6.26 & $1.1 \mathrm{E}-19$ & DPCR1 \\
\hline
\end{tabular}




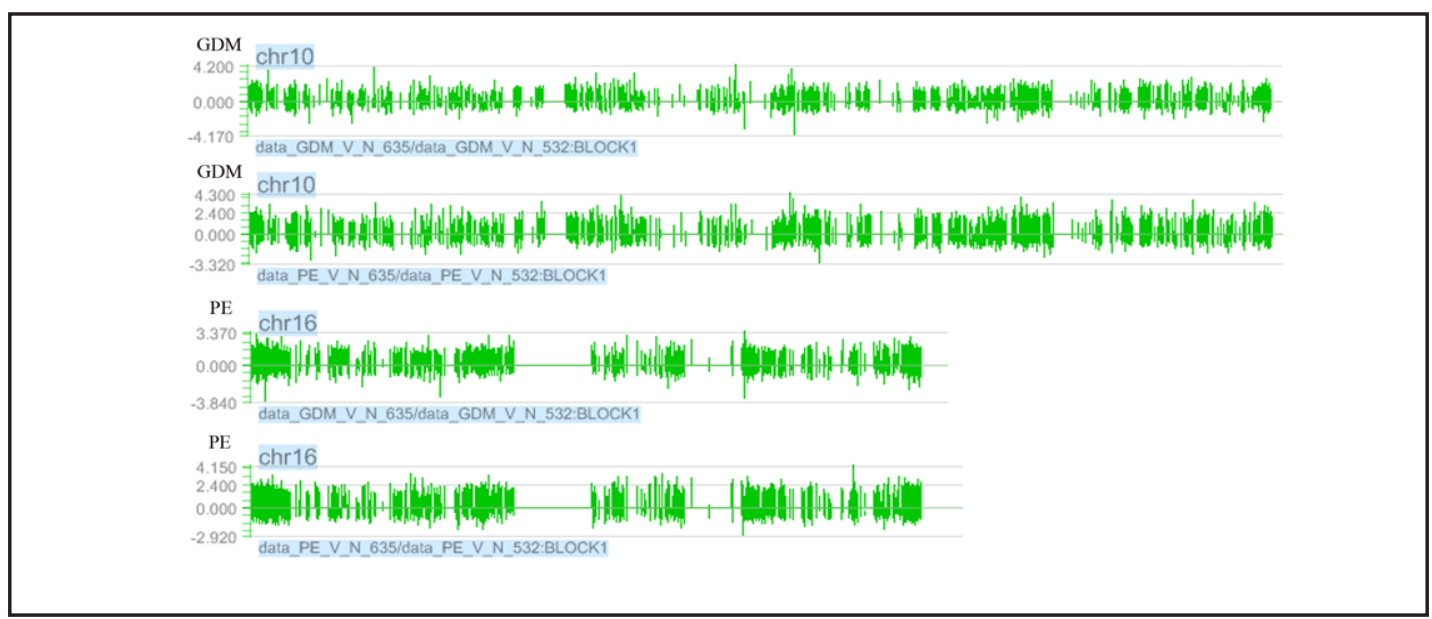

Fig. 2. Illustrative CpG methylation levels for GDM and PE in chromosomes 10 and 16.

Fig. 3. Gene ontology analysis of annotated genes showing differential methylation clustering by biological process: (A) functional categorization of PE, (B) functional categorization of GDM.

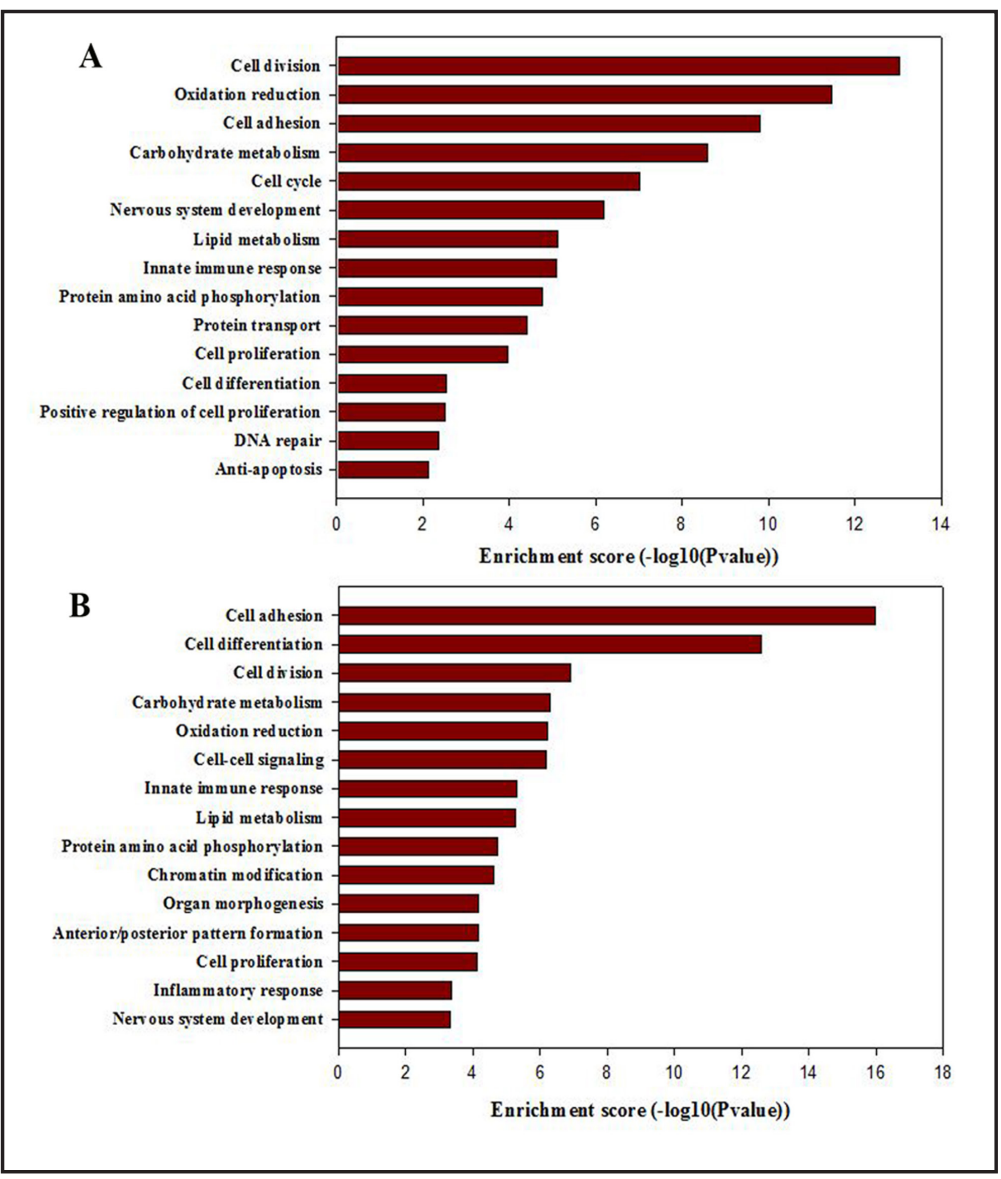

DNA methylation patterning in human placentas from PE and GDM patients

DNA methylation profiles were obtained on 85 human placenta samples using the NimbleGen DNA Methylation Analysis. We first compared the methylation profiles between PE and GDM to investigate whether placentas could represent a good source from which to profile methylation changes. We detected 8191 locis with differential methylation in placentas from PE patients and 10,424 locis in placentas from GDM cases (Table 2). Further analysis showed that the overall methylation patterns of PE and GDM were similar, with more than $64.4 \%$ (3348/5195) of the total autosomal annotated genes reported exhibiting consistent methylation (Fig. 1A). 
Fig. 4. Pathway analysis of annotated genes with methylation changes in PE and GDM patients compared with control: (A) functional categorization of annotated genes from PE group, (B) functional categorization of annotated genes from GDM group.

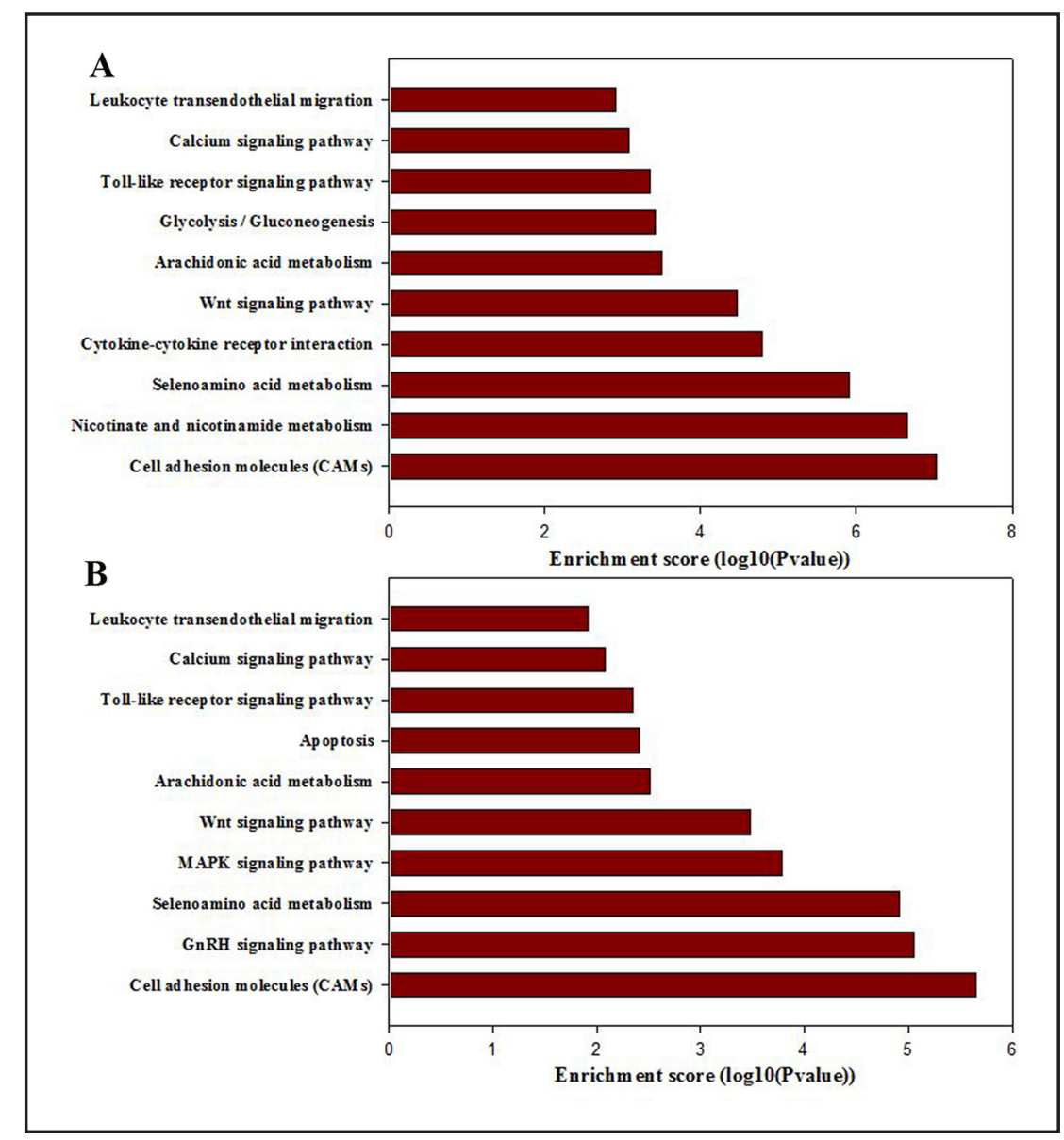

We assigned those regions exhibiting consistent methylation into distinct categories of genomic elements and then analyzed the Methyl-seq data in each sample according to each type of functional element. The annotated genomic elements are (1) promoters, (2) exons, (3) introns, and (4) intergenic regions. In Fig. 1B, we present the degree of DNA methylation for each genomic element in each sample. We observed that regions that include exons and intergenic region domains are more highly unmethylated (less than $16.8 \%$ of regions methylated), whereas regions including those occupied promoters and introns are more highly methylated (from $24.5 \%$ to more than $45.4 \%$ of the sites are methylated, Fig. 1B). Thus, different genomic functional elements vary in their degree of DNA methylation.

Further analysis revealed that these regions with differential methylation are spread throughout the genome and cover all chromosomes (see Fig. 1C). It is remarkable to see that chromosome 1 comprised the largest component of 10.5\% with 1118 (PE) and 827 (GDM). There were only 127 (PE) and 118 (GDM) regions on chromosome 13 (1.3\%). Illustrative CpG methylation levels for single chromosomes (chr 10 and chr 16) are shown in Fig. 2. As shown in Table 3, 52 hypomethylated regions constituted a significantly greater proportion of these regions; both changed more than threefold in PE and GDM patients, and 37 hypemethylated regions were present at a significantly higher level in PE and GDM patients (abundance changed more than fourfold). All regions involved showed statistically significant changes $(\mathrm{p}<0.05)$.

Concordant variation of differentially methylated loci in human placentas from PE and GDM patients

Differential methylation analysis applying the Illumina Custom model was performed after normalization to control probes present in the array and using the control group as reference. We identified 3394 probes in placentas from PE patients and 4021 in placentas 


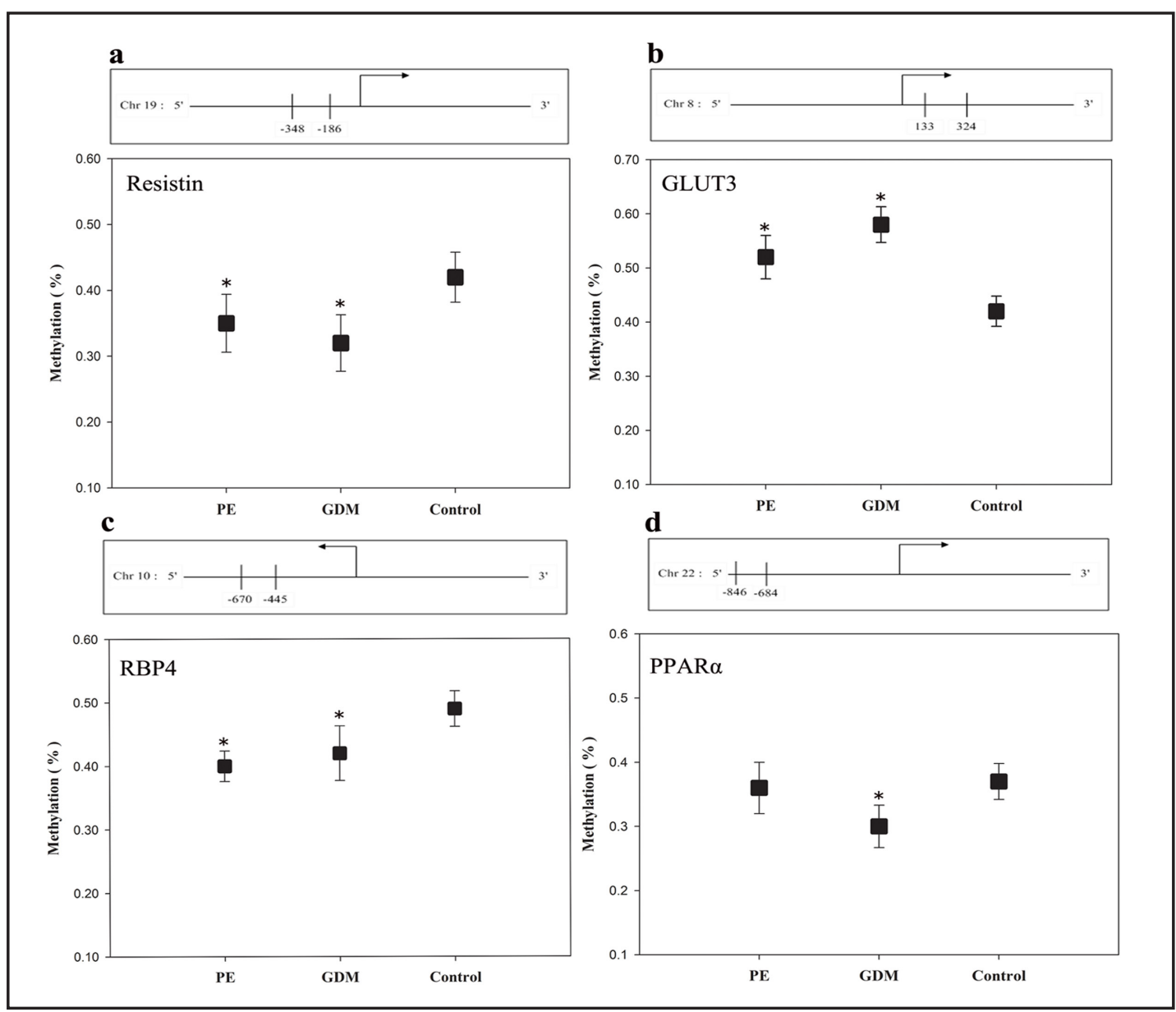

Fig. 5. Site-specific CpG-methylation analysis in selected genes. The percentage of DNA methylation was assessed with bisulfite sequencing for (A) resistin, (B) GLUT3, (C) RBP4 and (D) PPAR $\alpha$ in 27 placentas with PE, 28 placentas with GDM, and 30 normal placentas. P-values (indicated as (*’) were calculated using the Student's $t$-test. Locations for the differentially methylated regions targeted by bisulfite sequencing assays (regions bounded by vertical lines) are indicated in the box above the plots.

from GDM patients with increased methylation, whereas 4797 probes in placentas from PE patients and 6403 in placentas from GDM patients showed decreased methylation in PE (Table 2). We further refined our analysis to the fraction of autosomal probes showing differential methylation and an association with annotated genes, which corresponded to a total of 3878 genes in placentas from PE patients (1738 with increased and 2140 with decreased methylation) and 4656 annotated genes in GDM patients (2012 with increased and 2644 with decreased methylation, Table 2). Strikingly, 64.4\% (3348/5195) of the annotated genes with different methylation presented concordant changes in methylation between PE and GDM patients (1532 loci with increased methylation and 1816 with decreased methylation, respectively, Table 2).

\section{Gene Ontology and Pathway Analysis}

A gene-ontological term and canonical pathways investigation were designed to determine whether any common functional trends are associated with the genes exhibiting differences from PE and GDM groups compared with the control group. The top 15 GO categories for each diagnostic group can be observed in Fig. 3, which lists all significant GO categories with a $\mathrm{p}<0.05$. GO categories detected by this analysis include genes involved in the epigenetic regulation of transcription and development. The results showed that the 
same functional groups were affected in PE and GDM patients, with cell adhesion and cell division being the more populated clusters and including genes related to carbohydrate metabolism and lipid metabolism, molecular pathways largely implicated in PE and GDM in common.

Ingenuity Pathway Analysis was used to identify pathways and gene networks represented among the sets of protein-coding mRNAs identified in the VSD gene expression signature. In our survey of existing data, annotated genes that exhibited differences from GDM groups compared with the control group mainly involved the following pathways: (1) cell adhesion molecules (CAMs), (2) nicotinate and nicotinamide, (3) metabolism selenoamino acid metabolism, (4) cytokine-cytokine receptor interaction, (5) Wnt signaling pathway, (6) arachidonic acid metabolism, (7) glycolysis/gluconeogenesis, (8) toll-like receptor signaling pathway, (9) calcium signaling pathway, and (10) leukocyte transendothelial migration. The annotated genes exhibiting differences from GDM groups compared with the control group mainly involved the following pathways: (1) cell adhesion molecules (CAMs), (2) GnRH signaling pathway, (3) selenoamino acid metabolism, (4) MAPK signaling pathway, (5) Wnt signaling pathway, (6) arachidonic acid metabolism, (7) apoptosis, (8) toll-like receptor signaling pathway, (9) calcium signaling pathway, and (10) leukocyte transendothelial migration (Fig. 4).

\section{Site-Specific CpG-Methylation Analysis in Selected Genes}

The above analyses suggest that a number of methylation changes in placentas are shared between PE and GDM, positioning these genes (Table 2) that co-vary among tissues as candidates for biomarker discovery. To determine the potential association of these covarying genes between PE and GDM, we tested four genes' loci that have been confirmed as participating in carbohydrate metabolism or lipid metabolism to further verify the microarray approach. We quantitatively measured site-specific $\mathrm{CpG}$ methylation upstream of resistin ( $\mathrm{n}=15$ ), GLUT3 (glucose transporter $3, \mathrm{n}=15$ ), RBP4 (retinol binding protein $4, \mathrm{n}=$ 15), PPAR $\alpha$ (peroxisome proliferator-activated receptor alpha, $\mathrm{n}=15$ ). We focused primarily on surrounding $\mathrm{CpG}$ positions located in or near genomic regions corresponding to specific microarray probes.

Our site-specific CpG analyses show good consistency with data obtained from microarray analysis although the absolute differences observed are generally small. Two examples are shown in Fig. 5 for regions upstream of the genes' resistin and RBP4. Microarray analysis predicted these regions to be hypomethylated in PE and GDM samples. Fig. 5B shows BSP data confirming GLU3 hypermethylation both in PE and GDM samples compared with controls. BSP data also verified PPAR $\alpha$ hypomethylation in samples from women with GDM. However, there is no significant methylation difference between PE groups and control groups (Fig. 5D).

\section{Discussion}

Placental function is directly linked to fetal development and health, and as such, placental dysfunction is implicated in many pregnancy-associated diseases [18]. Accordingly, aberrant placental gene expression has been linked to pre-eclampsia, intrauterine growth restriction, gestational diabetes mellitus and gestational trophoblastic disease [19-25]. In this study, we performed a microarray-based epigenomic scan using human CpG-island plus Promoter microarrays and observed placenta-associated DNA methylation differences in numerous loci. Many genes that have been functionally linked to PE and GDM etiology were included. Consistent with increasing evidence, gestational diabetes mellitus and preeclampsia have several mechanisms in common. We identified epigenetic changes in lociassociated pathophysiologies of both illnesses with similar methylation patterns.

We identified 3394 probes in placentas from PE patients and 4021 in placentas from GDM patients with increased methylation whereas 4797 probes in placentas from PE 
patients and 6403 in placentas from GDM patients showed decreased methylation in PE (Table 2). We further refined our analysis to the fraction of autosomal probes showing differential methylation and associated with annotated genes, which corresponded to a total of 3878 genes in placentas from PE patients and 4656 annotated genes in GDM patients. Strikingly, 64.4\% (3348/5195) of the annotated genes with different methylation presented concordant changes in methylation between PE and GDM patients (Table 2). Consistent with previous reports, although PE and GDM have distinctive clinical and pathophysiologic characteristics, they share some important similarities: both conditions develop during pregnancy, and the clinical syndromes disappear after delivery. Many risk factors such as obesity, elevated blood pressure, dyslipidemia, insulin resistance, and hyperglycemia are associated with both PE and GDM; and patients with a history of PE or GDM have increased risk of developing cardiovascular disease compared to women without such a history [1-22].

Several interesting GO categories are highlighted by our analysis, including several involved in various epigenetic processes, transcription, and development. The top 15 GO categories for each diagnostic group can be observed in Fig. 2, which lists all significant GO categories with a $p<0.05$. Our results show that the same functional groups were affected in PE and GDM patients, with cell adhesion and cell differentiation being the more populated clusters (Fig. 2); including genes related to carbohydrate metabolism and lipid metabolism, the molecular pathways of PE and GDM are largely implicated in common. Our findings may represent alterations to such pathways related to PE and GDM, potentially linking the two pathological processes with carbohydrate metabolism and lipid metabolism pathways. Additional studies are warranted to expand on these examinations in later life endpoints to clarify the biological mechanisms at play.

It is important to note that we cannot definitively determine if these altered profiles of DNA methylation are a response of the placenta to the intrauterine environment and an independent association between these specific profiles of DNA methylation. Ongoing research is now revealing that gene-regulating methylation events may occur in regions outside of $\mathrm{CpG}$ islands such as the region of the regulated gene [32]. More comprehensive approaches including more inclusive arrays and genome-wide sequencing will be required to fully identify all regions contributing to PE and GDM

We focused our investigation on methylation patterning because of the highly stable nature of DNA methylation marks. Previous studies have shown that labor induces altered expression of genes in the human placenta [33], and although the methylation status is stable, the corresponding gene expression may not be [34]. Therefore, we believe that examination of methylation may reflect changes occurring over the course of in utero development and not only occurring in the final moments of pregnancy and delivery. We used a relatively unbiased, genome-wide method to identify locis whose methylation status in the placenta was most associated with PE and GDM.

Microarray analyses suggest that a number of methylation changes in the placenta are shared between PE and GDM, positioning these genes (Table 2) that co-varied among tissues as candidates for biomarker discovery. To determine the potential association of those genes co-varying between PE and GDM, we tested four gene loci that have been confirmed as participating in carbohydrate metabolism and lipid metabolism to further verify the microarray approach. We quantitatively measured site-specific CpG methylation upstream of resistin, GLUT3, RBP4 and PPAR $\alpha$. GLUT3 as a high-affinity transporter has been implicated in the extraction of glucose from the fetal circulatory system for storage as glycogen in the placental endothelial cells [35]. GLUT3 upregulation in maternal diabetes is consistent with elevated glycogen depots in the placental endothelium in this condition. Aberrant GLUT3 expression was therefore suggested as contributing to fetal hyperglycemia in maternal diabetes [36]. It may likewise play a role in integrating environmental signals, resulting in altered placental function and GDM. Increased circulating RBP4 levels have been reported in several metabolic complications including obesity, insulin resistance, polycystic ovary syndrome and cardiovascular disease [37]. RBP4 is a physiological constituent of amniotic fluid [38]. Thus, DNA methylation of RBP4 target genes may affect the maternal- 
fetal interface, resulting in PE and GDM.

In conclusion, we report genome-wide alterations in DNA methylation associated with PE and GDM pathology in placentas. Although there are many differences in pathogenesis, we detected similar methylation patterns in both tissues. Significantly, we identified a set of genes whose methylation coordinately varies in PE and GDM placentas, with the majority of these loci previously being implicated in both pathologies. This study will be helpful in exploring gestational diabetes mellitus and pre-eclampsia's having several mechanisms in common from the perspective of epigenetics.

\section{Acknowledgments}

This study was supported by grants from the National Natural Science Foundation of China (No. 81200442), the Natural Science Foundation of Jiangsu Province, China (No. BK2011106); the Scientific and Technological Personnel Start Projects Foundation of the Nanjing Health Bureau, Jiangsu Province, China (No. QYK11141); and the Selected Member of Nanjing Health Youth Talent Training Project (QRX11209).

\section{References}

1 Alwan N, Tuffnell DJ, West J: Treatments for gestational diabetes mellitus. Cochrane Database Syst Rev 2009;8:CD003395.

2 Sibai BM: American College of Obstetricians and Gynecologists. Hypertension in Pregnancy 1996; Technical Bulletin No. 219. Washington, DC: ACOG.

3 Östlund I, Haglund B, Hanson U: Gestational diabetes mellitus and Pre-eclampsia. Eur J Obstet Gynecol Reprod Biol 2004;113:12-16.

4 Wendland EM, Duncan BB, Belizan JM, Vigo A, Schmidt MI: Gestational diabetes mellitus and preeclampsia: common antecedents? Arq Bras Endocrinol Metabol 2008;52:975-984.

5 Vambergue A, Nuttens MC, Goeusse P, Biausque S, Lepeut M, Fontaine P: Pregnancy induced hypertension in women with gestational carbohydrate intolerance: the diagest study. Eur J Obstet Gynecol Reprod Biol 2002;102:31-35.

-6 Schneider S, Freerksen N, Maul H, Roehrig S, Fischer B, Hoeft B: Risk groups and maternal-neonatal complications of Pre-eclampsia-Current results from the national German Perinatal Quality Registry. J Perinat Med 2011;39:257-265.

7 Crowther CA, Hiller JE, Moss JR, McPhee AJ, Jeffries WS, Robinson JS: Effect of treatment of gestational diabetes mellitus on pregnancy outcomes. N Engl J Med 2005;352:2477-2486.

Solomon CG, Seely EW: Pre-eclampsia-searching for the cause. N Engl J Med 2004;350:641-642.

Roberts JM, Gammill H: Insulin resistance in Pre-eclampsia. Hypertension 2006;47:341-342.

Bryson CL, Ioannou GN, Rulyak SJ, Critchlow C: Association between gestational diabetes mellitus and pregnancy-induced hypertension. Am J Epidemiol 2003;158:1148-1153.

Nakao M: Epigenetics: interaction of DNA methylation and chromatin. Gene 2001;278:25-31.

Holliday R: The inheritance of epigenetic defects. Science 1987;238:163-170.

Reik W, Dean W: DNA methylation and mammalian epigenetics. Electrophoresis 2001;22:2838-2843.

Bird A: DNA methylation patterns and epigenetic memory. Genes Dev 2002;16:6-21.

Bird AP: CpG-rich islands and the function of DNA methylation. Nature 1986;321:209-213.

Benirschke K, Kaufmann P: Pathology of the human placenta. New York, Springer-Verlag 2000, pp 281-297.

Sobrevia L, Abarzua F, Nien JK, Salomon C, Westermeier F, Puebla C: Review: differential placental

macrovascular and microvascular endothelial dysfunction in gestational diabetes mellitus. Placenta

2011;32:59-64. 
18 Kang JH, Song H, Yoon JA, Park DY, Kim SH, Lee KJ: Pre-eclampsia leads to dysregulation of various signaling pathways in placenta. J Hypertens 2011;29:928-936.

19 Holdsworth-Carson SJ, Lim R, Mitton A, Whitehead C, Rice GE, Permezel M: Peroxisome proliferatoractivated receptors are altered in pathologies of the human placenta: gestational diabetes mellitus mellitus, intrauterine growth restriction and Pre-eclampsia. Placenta 2010;31:222-229.

20 Hoegh AM, Borup R, Nielsen FC, Sorensen S, Hviid TV: Gene expression profiling of placentas affected by pre-eclampsia. J Biomed Biotechnol 2010;2010:787545.

21 Fulop V, Mok SC, Berkowitz RS: Molecular biology of gestational trophoblastic neoplasia: a review. J Reprod Med 2004;49:415-422.

22 Founds SA, Conley YP, Lyons-Weiler JF, Jeyabalan A, Hogge WA, Conrad KP: Altered global gene expression in first trimester placentas of women destined to develop Pre-eclampsia. Placenta 2009;30:15-24.

$\checkmark 23$ Dieber-Rotheneder M, Beganovic S, Desoye G, Lang U, Cervar-Zivkovic M: Complex expression changes of the placental endothelin system in early and late onset Pre-eclampsia, fetal growth restriction and gestational diabetes mellitus. Life Sci 2012;9:710-715.

24 Myatt L: Placental adaptive responses and fetal programming. J Physiol 2006;572:25-30.

25 Burton GJ, Jauniaux E, Charnock-Jones DS: The influence of the intrauterine environment on human placental development. Int J Dev Biol 2010;54:303-312.

26 Yuen RK, Avila L, Penaherrera MS, von Dadelszen P, Lefebvre L, Kobor MS: Human placental-specific epipolymorphism and its association with adverse pregnancy outcomes. PloS One 2009;4:e7389.

27 Ching T, Song M-A, Tiirikainen M, Molnar J, Berry M, Towner D, Garmire LX: Genome-wide hypermethylation coupled with promoter hypomethylation in the chorioamniotic membranes of early onset pre-eclampsia. Mol Human Reprod 2014:20;885-904.

28 Lash G, Burton G, Chamley L, Clifton V, Constancia M, Crocker I, Dantzer V, Desoye G, Drewlo S, Hemmings DG, Hiendleder S, Kalionis B, Keelan JA, Kudo Y, Lewis RM, Manuelpillai U, Murthi P, Natale D, Pfarrer C, Robertson S, Saffery R, Saito S, Sferruzzi-Perri A, Sobrevia L, Waddell BJ, Roberts CT: IFPA meeting 2009 workshops report. Placenta 2010;31:S4-S20.

29 Rauch TA, Pfeifer, GP: The MIRA method for DNA methylation analysis. Methods Mol Biol 2009;507:65-75.

-30 Rauch, TA, Wu X, Zhong X, Riggs AD, Pfeifer GP: A human B cell methylome at 100-base pair resolution. Proc Nat Acad Sci USA 2009;106:671-678.

-31 Han T, Wang J, Tong W, Moore MM, Fuscoe JC, Chen T: Microarray analysis distinguishes differential gene expression patterns from large and small colony Thymidine kinase mutants of L5178Y mouse lymphoma cells. BMC Bioinformatics 2006; 7:S9.

- 32 Irizarry RA, Ladd-Acosta C, Wen B, Wu Z, Montano C, Onyango P: The human colon cancer methylome shows similar hypo-and hypermethylation at conserved tissue-specific CpG island shores. Nat Genet 2009;41:178-186.

33 Lee K, Shim S, Kang K, Kang J, Park D, Kim S: Global gene expression changes induced in the human placenta during labor. Placenta 2010;31:698-704.

-34 Avila L, Yuen RK, Diego-Alvarez D, Penaherrera MS, Jiang R, Robinson WP: Evaluating DNA methylation and gene expression variability in the human term placenta. Placenta 2010; 31:1070-1077.

-35 Hahn D, Blaschitz A, Korgun ET, Lang I, Desoye G, Skofitsch G: From maternal glucose to fetal glycogen: expression of key regulators in the human placenta. Mol Hum Reprod 2001;7:1173-1178.

- 36 Desoye G, Korgun ET, Ghaffari-Tabrizi N, Hahn T: Is fetal macrosomia in adequately controlled diabetic women the result of a placental defect? A hypothesis. J Matern Fetal Neonatal Med 2002;11:258-261.

37 Esteve E, Ricart W, Fernandez-Real JM: Adipocytokines and insulin resistance: the possible role of lipocalin-2, retinol binding protein-4, and adiponectin. Diabetes Care 2009;32:362-327.

- 38 Vaisbuch E, Mazaki-Tovi S, Kusanovic JP: Retinol binding protein 4: an adipokine associated with intraamniotic infection/inflammation. J Matern Fetal Neonatal Med 2010;23:111-119. 